\title{
Place de la tomographie volumique à faisceau conique en imagerie dento-maxillofaciale
}

\section{RÉSUMÉ}

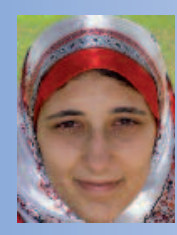

Laila BENJELLOUN

Ancienne interne au centre de consultation et de soin dentaire de Rabat, Maroc,

Centre hospitalier Ibn Sina,

Résidente

au service d'odontologie chirurgicale au centre de consultation et de soin dentaire de Rabat, Maroc.

Secteur 9, Rés. Zahrat Riad, Imm. D, Appt D10, Hay Riad, Rabat, Maroc.

\section{Karima EL HARTI}

Professeur assistante

en odontologie chirurgicale

Faculté de médecine dentaire de Rabat,

Centre de consultation et de soin dentaire de Rabat, Maroc.

\section{Wafaa EL WADY}

Professeur de l'enseignement supérieur en odontologie chirurgicale

Faculté de médecine dentaire de Rabat, Chef de service d'odontologie chirurgicale au centre de consultation et de soin dentaire de Rabat, Maroc,

Centre hospitalier Ibn Sina.
La tomographie volumique à faisceau conique ou plus communément appelée « cone beam » est une nouvelle technique d'imagerie qui permet de visualiser l'ensemble du complexe maxillofacial.

Ses performances techniques et dosimétriques, ainsi que son coût faible par rapport à la tomodensitométrie, peuvent la proposer comme alternative à cette dernière, notamment dans certaines indications cliniques, telles que les tumeurs odontogènes, les traumatismes maxillofaciaux, l'implantologie, le rapport des dents de sagesse avec les structures avoisinantes...

La diffusion de cette technique, qui s'avère prometteuse, est déjà largement avancée. II est donc désormais important de faire le point sur la place réelle de cette technologie en imagerie dento-maxillofaciale.

\section{(C) EDP Sciences}




\section{Introduction}

$>$

La pratique du scanner dentaire (denta-

scanner), longtemps considérée comme

l'examen radiologique de référence en odontostomatologie, est aujourd'hui remise en question par l'arrivée sur le marché d'une technique concurrente, réputée moins irradiante : la tomographie volumique à faisceau conique (TVFC) ou plus communément appelée " cone beam » [1]. En anglais : cone beam computerized tomography (CBCT).

Introduite en Amérique du Nord en 2001, la TVFC devient de plus en plus utilisée dans le domaine dentaire. Grâce à la conicité du faisceau à rayons $X(R X)$, à la rotation rapide de $180^{\circ}$ ou $360^{\circ}$ du tube à $\mathrm{RX}$ et à un détecteur

\section{Historique}

La TVFC a été construite en premier lieu en 1982 à la clinique de Mayo pour des applications en angiographie. Les systèmes TVFC ont aussi été utilisés pour la radiothérapie, la mammographie et en peropératoire lors de chirurgies otorhinolaryngologiques [9].

Bien que l'équipement de la TVFC existe depuis un quart de siècle, ce n'est qu'au cours de cette dernière décennie qu'il a été possible de produire des systèmes moins coûteux et moins encombrants, utilisables par le chirurgien-dentiste dans son cabinet [9].

Le premier système TVFC commercialisé pour l'imagerie orale et maxillofaciale a été le NewTom ${ }^{\circledR}$, qui a été approuvé en premier lieu par la FDA (Food and Drug Administration) en avril 2001, et qui en est actuellement à sa qua- digital, la TVFC procure immédiatement une image radiographique bidimensionnelle ou tridimensionnelle d'une structure anatomique limitée ou de l'ensemble du complexe maxillofacial [11].

Cette nouvelle technique permet, pour un certain nombre d'indications, d'éviter un examen tomodensitométrique irradiant, tout en conservant les avantages de l'imagerie volumétrique [21].

Cet article rappelle les nombreux intérêts de la nouvelle technique d'imagerie volumique et explore ses différentes applications dans le domaine d'odontologie, tout en ayant pour but de l'appliquer selon des bases d'evidence based.

trième génération NewTom VG ${ }^{\circledR}$. Depuis, de nombreux systèmes ont été approuvés ou sont en développement. Ces derniers peuvent être divisés en trois groupes:

- les TVFC capables d'explorer une grande portion maxillofaciale et craniale avec une seule exposition (FOV large) (FOV = field of view ou champ de vue) ;

- des TVFC avec un FOV moins large ou à champ réduit. Le modèle de référence est I'Accuitomo ${ }^{\circledR}$ qui explore un volume réduit de I'arcade, utilisé en endodontie ;

- le panoramique digital hybride/systèmes TVFC incluant des détecteurs séparés pour les deux fonctions. Quelques-uns de ces derniers peuvent aussi procurer une option de céphalogramme digital 2D [9]. 


\section{Principe de la technique « cone beam »}

La technique cone beam repose sur le principe de tomosynthèse développé par Ziedes Des Plantes et Grant au début des années 1970 [5, 12] (fig. 1 et 2).

Le cone beam consiste en un générateur de rayons $\mathrm{X}$ qui émet un faisceau de forme conique. La conicité du faisceau constitue la principale particularité du système et diffère du faisceau de rayon $\mathrm{X}$ mince caractérisant la tomodensitométrie (fig. 3).

Le faisceau traverse l'objet à explorer, il est ensuite analysé, après atténuation, par un système de détection qui peut comprendre soit un capteur plan (Accuitomo, I-Cat), soit un amplificateur de brillance (Galileos, NewTom 3G) [5, 12]. Alors que le cone beam ne peut utiliser qu'un seul capteur pour acquérir les images, le scanner nécessite un ensemble de détecteurs [5].

Le tube à rayons $X$ et l'aire de détecteurs sont solidaires et alignés. Ils réalisent autour du sujet une seule rotation qui peut être complète $\left(180^{\circ}\right.$ à $360^{\circ}$ ) et qui permet d'acquérir des données numériques en $2 \mathrm{D}$ dans les différents plans de l'espace, c'est-à-dire qu'il permet d'obtenir des images planes numérisées se répartissant selon

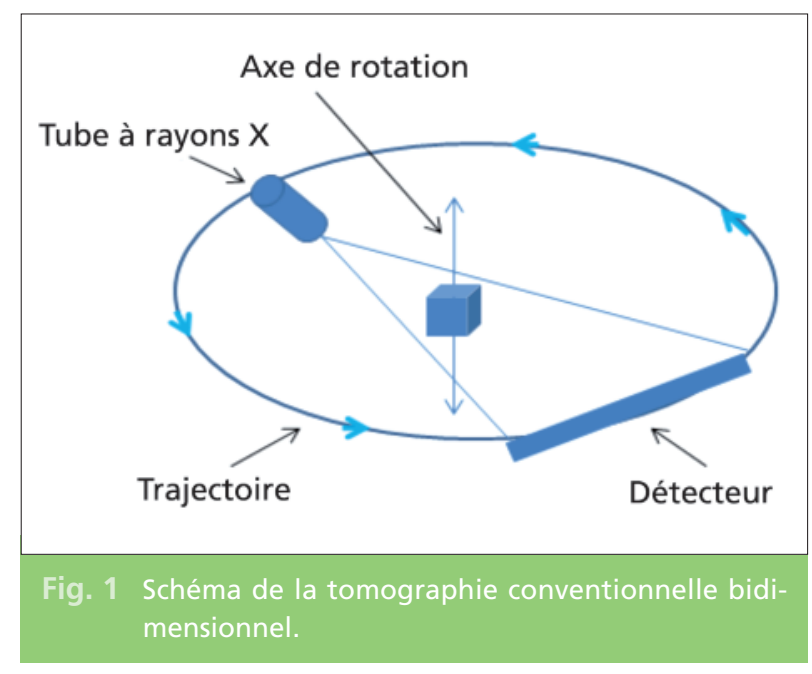

la trajectoire circulaire de rotation du système. Ces données numériques sont ensuite transmises à un ordinateur et traitées par des algorithmes de reconstruction volumique d'un cube contenant l'objet. Le volume étudié est composé de voxels dont le côté est de la taille d'un pixel, mesuré en mm [5, 12] (fig. 4).

À partir de ce volume numérisé, seront élaborées les reconstructions $2 \mathrm{D}$ et $3 \mathrm{D}$. Les reconstructions 2D seront, comme pour le scanner, coronales, sagittales, obliques, panoramiques (comparables aux reconstructions du Dentascan) et restituées en taille réelle $[5,12]$.

Les appareils se distinguent donc du scanner traditionnel qui effectue des coupes linéaires se superposant lors des multiples rotations du système. Suivant sa précision (résolution) et le champ que l'on souhaitera radiographier, il faudra effectuer un certain nombre de coupes pour un examen scanner. Le cone beam quant à lui, travaille non plus avec un faisceau RX mince, mais avec un faisceau ouvert, conique ; ce qui lui permettra en une seule révolution de balayer l'ensemble de la zone à radiographier [12].

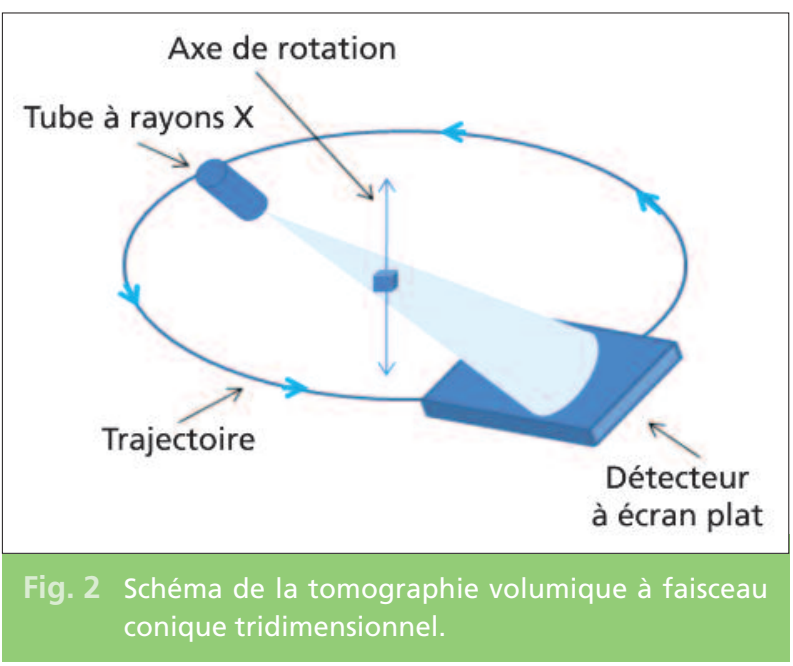




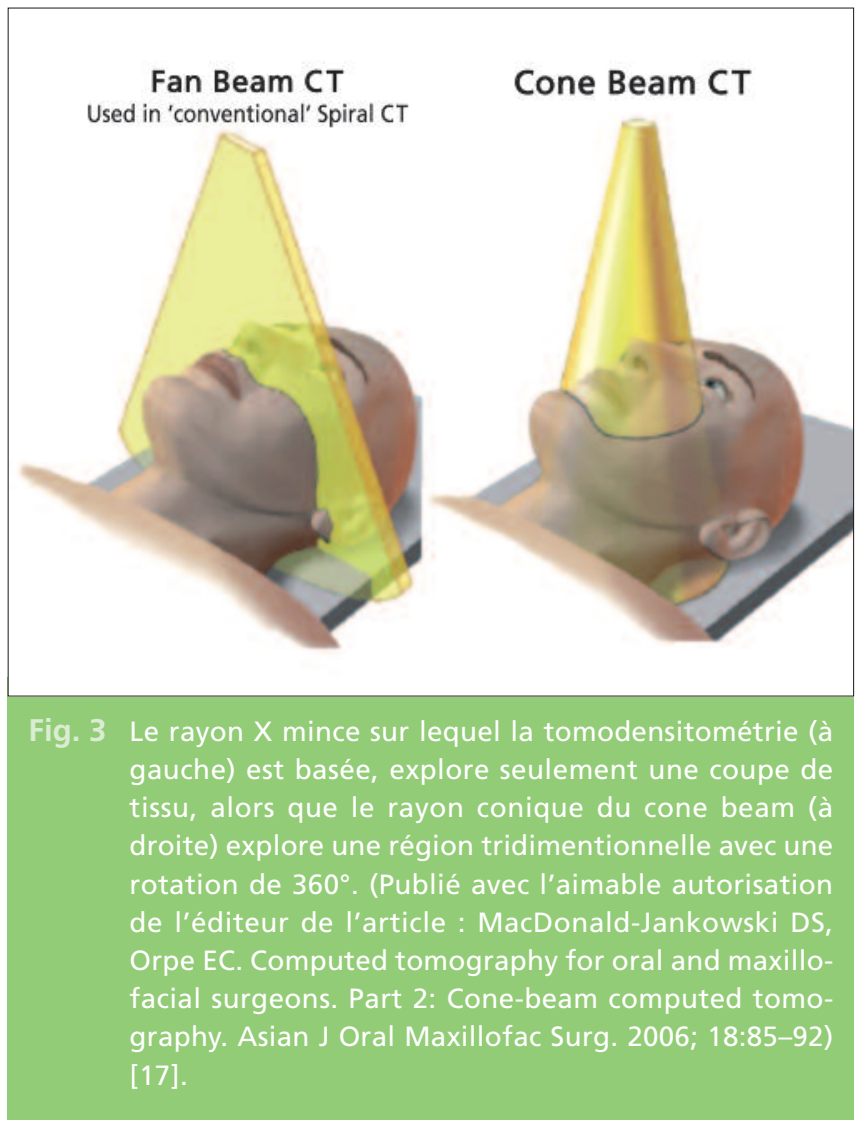

Les informations obtenues pourront ensuite être transmises par l'intermédiaire de fichiers DICOM (Digital Imaging and Communications in Medicine - National Electrical Manufacturers Association, Rosslyn, Virginie) soit pour l'archivage, soit à un tiers parti afin d'obtenir un diagnostic plus poussé ou pour la fabrication de guides chirurgicaux par stéréolithographie avec des logiciels comme le SimPlant ${ }^{\circledR}$, le

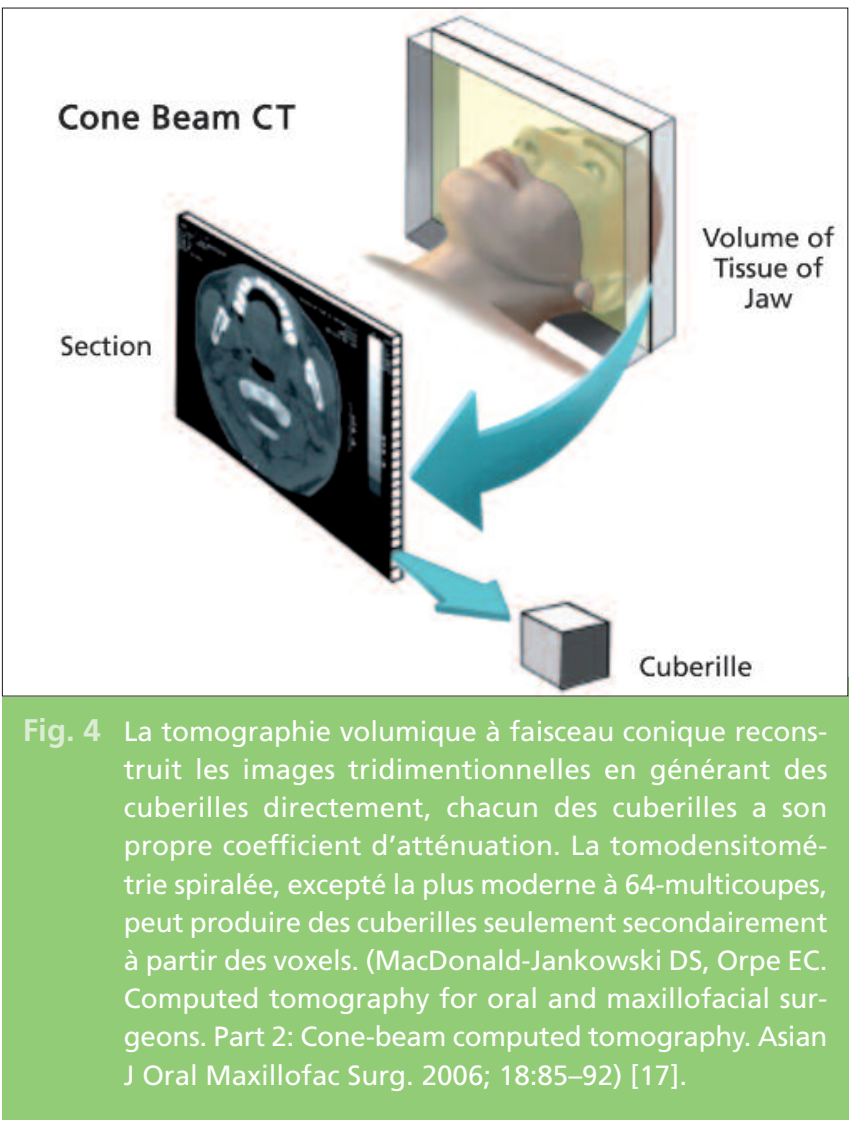

NobelGuide ${ }^{\circledR}$, I’EasyGuide ${ }^{\circledR}$, I'ImplantMaster ${ }^{\circledR}$ [8].

Pour réaliser l'examen, le sujet est confortablement placé en décubitus dorsal sur la table motorisée, ou bien il est le plus souvent assis, sa tête étant placée dans un système de contention. Le patient doit rester immobile en évitant de déglutir durant l'examen [21].

\section{Champs d'application cliniques du « cone beam »}

La TVFC peut fournir les informations 3D essentielles sur les structures dentaires et maxillofaciales pour le diagnostic et la planification de traitement de beaucoup de procédures telles que la pose des implants dentaires, I'évaluation de l'étendue des fractures maxillo-faciales, les 
chirurgies orthognathiques, les pathologies tumorales, la relation spatiale entre les troisièmes molaires et le canal mandibulaire, l'évaluation de la croissance et du développement des maxillaires pour la planification orthodontique, les parois sinusales avant la greffe osseuse... [8].

\section{Implantologie}

et chirurgie buccale

L'Académie américaine de radiologie buccale et maxillofaciale recommande l'utilisation de la TVFC dans le plan de traitement préimplantaire [24].

L'avantage de la TVFC dans la visualisation tridimensionnelle de l'alvéole et la possibilité de faire des mesures avant une chirurgie implantaire, semble évident dans le domaine de l'implantologie [20]. Cela permet d'avoir des informations sur la hauteur et la largeur osseuse, la position du nerf mandibulaire, ainsi qu'une mesure objective de la qualité de l'os. La TVFC permet donc au chirurgien d'avoir la possibilité d'anticiper la mise en place de l'implant et même de le placer dans un modèle virtuel $[1,20]$.

Certains auteurs recommandent également I'utilisation de la TVFC pour l'évaluation préopératoire des cas compliqués des dents de sagesse mandibulaires [22].

Des études in vivo ont également mis en évidence la qualité diagnostique satisfaisante de la TVFC pour préciser la position d'éléments anatomiques, par exemple :

- la topographie du canal mandibulaire avant la pose d'implants ou avant l'extraction chirurgicale de troisièmes molaires inférieures incluses $[8,13]$;

- ou encore la visualisation des sinus maxillaires avant la pose de greffe osseuse lors des élévations sinusales $[1,8]$.
La TVFC réduira ainsi le nombre de complications sérieuses telles qu'une lésion du nerf mandibulaire [16].

La TVFC peut également aider le clinicien à évaluer la position des dents incluses, l'éruption tardive de dents par rapport aux dents adjacentes et de planifier un plan de traitement [8, 10, 24]. Une étude de Bjerklin et al. en 2006 [2] a démontré qu'avec I'utilisation de la TVFC, les cliniciens, dans plus de la moitié des cas incluant un diagnostic de canines incluses, avaient dû changer leur plan de traitement [2].

La TVFC pourrait donc se substituer au scanner pour l'analyse préimplantaire ainsi que pour le bilan préchirurgical avant l'extraction de dents incluses et surtout des troisièmes molaires mandibulaires [18], en complément d'un premier bilan radiographique insuffisamment contributif. Cette décision doit toutefois s'appuyer sur un principe de radioprotection en choisissant un appareil, une taille de champ et un paramétrage qui, pour une qualité d'image comparable et suffisante, induisent un niveau d'irradiation inférieur à celui d'un examen scanner [11].

\section{Chirurgie maxillofaciale}

Lorsqu'une imagerie sectionnelle est nécessaire en chirurgie orthognatique, en traumatologie, pour les pathologies osseuses, le CBCT pourrait se substituer au scanner dans des cas sélectionnés à condition :

- qu'une exploration des tissus mous ne soit pas requise ;

- que le niveau d'irradiation soit moindre que celui obtenu avec l'imagerie scanner, au regard du principe de radioprotection et selon des règles d'optimisation.

Toutefois, des études complémentaires contrôlées sont nécessaires pour en évaluer les bénéfices potentiels [11, 20]. 


\section{Endodontie}

\section{et chirurgie endodontique}

L'imagerie TVFC en endodontie peut présenter un intérêt dans certains cas bien sélectionnés, lorsque les informations fournies par la clinique et la radiologie conventionnelle ne sont pas suffisamment contributives au diagnostic et qu'une image tridimensionnelle est indispensable $[8,11$, 25]. C'est le cas :

- d'un bilan péri-apical préchirurgical, particulièrement dans la région maxillaire postérieure ou dans la région du foramen mentonnier ;

- de la recherche et de la localisation d'un canal radiculaire supplémentaire ;

- d'un bilan d'une pathologie radiculaire type fracture et résorption $[3,8,11,25]$.

L'exploration endodontique exige une TVFC avec une forte résolution spatiale pour obtenir une plus grande exactitude et, par conséquent, un diagnostic plus précis permettant parfois d'éviter au patient un traitement inapproprié $[8,11,25]$.

Cependant, le recours à l'imagerie TVFC doit être justifié par l'amélioration, dans ces cas précis, de la prise en charge et le pronostic de la dent [11].

\section{Parodontologie}

L'imagerie $C B C T$ en parodontologie peut présenter un intérêt dans un très petit nombre de cas sélectionnés, tels que le bilan préchirurgical pour le traitement de lésions de la furcation de molaires, en apportant des informations non fournies par la clinique et la radiologie conventionnelle (informations anatomiques type fusion ou proximité radiculaire) [11].

\section{Orthodontie}

Les mesures céphalométriques obtenues avec la TVFC sont comparables à celles obtenues avec la céphalométrie conventionnelle et sont pour la plupart suffisamment précises et reproductibles [19]. Toutefois, des études supplémentaires sont nécessaires afin d'évaluer les bénéfices cliniques de la TVFC pour l'analyse céphalométrique.

Par ailleurs, au regard du principe de radioprotection et selon des règles d'optimisation, la TVFC pourrait se substituer au scanner quand une analyse tridimensionnelle est indispensable, par exemple pour des cas de dysmorphoses maxillofaciales majeures nécessitant une approche chirurgicale [11, 25].

\section{Pathologies de l'articulation temporo-mandibulaire (ATM)}

Des études (in vitro) soulignent la faisabilité technique de l'examen cone beam pour l'étude des structures osseuses de I'ATM, notamment pour la mise en évidence d'ostéophytes, d'érosions, de fractures, d'ankyloses, et de tumeurs... $[8,13]$.

Toutefois les indications restent limitées à la traumatologie et aux pathologies dégénératives, les pathologies discales et ligamentaires étant quant à elles explorées par l'imagerie par résonance magnétique $[8,11]$.

\section{Otorhinolaryngologie} (ORL)

Des études préliminaires soulignent la faisabilité technique de l'examen cone beam pour explorer les sinus, pour la navigation chirurgicale avec imagerie peropératoire en chirurgie endoscopique du sinus ainsi que pour évaluer des pathologies et des implants au niveau de l'oreille moyenne.

Le TVFC pourrait se substituer au scanner pour le bilan des sinusites du fait de son intérêt dosimétrique [11]. Des études supplémentaires sont toutefois nécessaires pour confirmer ces premiers résultats [11]. 


\section{Principaux avantages de la TVFG}

L'utilisation de la TVFC en pratique clinique procure de nombreux avantages comparés au scanner.

\section{Dosimétrie réduite}

L'avantage principal de la TVFC par rapport au scanner est la réduction de la dose de radiation reçue par le patient [23]. Cet avantage trouve son bénéfice maximal dans I'utilisation chez I'enfant [8, 21, 23].

Des enquêtes ont montré que la dose efficace (dose permettant de calculer le risque statistique théorique maximum d'apparition d'effets cancérigènes) [1] mesurée pour une TVFC est de $13 \mu \mathrm{Sv}$ à $82 \mu \mathrm{Sv}$, alors que pour un scanner multicoupes, elle est de $474 \mu S v$ à $1160 \mu S v$, et pour un orthopantomogramme, de 3 à $24 \mu S v[14,15]$. Avec un même appareil cone beam, les doses peuvent varier selon le volume exploré et les paramètres techniques retenus. Elles seront d'autant moins élevées que le champ exploré est réduit et les paramètres abaissés. De la même façon, certains appareils programmés sur mode ultra (haute résolution et champ maximal) procurent des doses proches des scanners optimisés $[5,11]$.

Cependant, les résultats d'une revue de De Vos et al. en 2009 ont montré qu'il n'y a pas encore des données basées sur l'evidence based concernant la dose d'irradiation provoquée par un appareil cone beam, et donc des études sont encore nécessaires dans ce domaine [6].

\section{Reconstructions}

\section{et travail de l'image}

La TVFC permet d'obtenir toutes les reconstructions réalisables par tomodensitométrie, mais avec des principes totalement différents.
Le fenêtrage s'effectue sur les reconstructions primaires, qui correspondent à des coupes axiales. Celles-ci peuvent être choisies selon n'importe quelle angulation sur le Scout view car l'acquisition des données se fait pour un volume complet, ce qui n'est pas le cas avec le scanner [21].

Différents types d'images en deux dimensions sont donc réalisables à partir d'une coupe axiale de référence : reconstructions transversales ou coronales, longitudinales curvilignes, axiales, frontales...

Les paramètres de l'image sont modifiables au niveau des consoles secondaires de manière à préciser une structure anatomique. De plus, le logiciel peut être muni d'un marqueur qui permet à l'opérateur d'effectuer des tracés sur toutes les coupes et, en particulier, sur les reconstructions longitudinales curvilignes, qui seront automatiquement transcrits sur toutes les autres coupes. Cette fonction permet de compenser le manque de définition parfois rencontré sur les reconstructions transversales, en repérant préalablement les structures utiles au praticien sur des coupes plus démonstratives [21].

Des améliorations ont été également faites, incluant un agrandissement, un fenêtrage, la possibilité d'ajouter des notes, et la mesure d'algorithmes (l'amélioration des algorithmes est capable d'optimiser l'apparence de l'image) [9].

\section{Résolution de l’image et précision des mesures}

L'élément de base pour les appareils cone beam, qui permet de définir la résolution spatiale, est un voxel isotropique, c'est-à-dire un cube. Ceci permet d'obtenir une image iden- 
tique dans tous les plans de l'espace. Dans le cas du scanner, le voxel est qualifié d'anisotropique, car il est représenté par un rectangle en volume. Dans ce cas, lors des différentes rotations dans les trois plans de l'espace, nous pouvons avoir des imprécisions sur la localisation d'un élément anatomique [9, 21].

La taille du voxel est aussi plus faible que celle d'un scanner (variant de 0,07 à $0,21 \mathrm{~mm}$ ) $[9,21]$. La précision des mesures effectuées est donc de l'ordre du dixième de millimètre, ce qui demeure relativement précis. La TVFC a une résolution spatiale et une fiabilité qui semblent comparables à celles du scanner pour explorer des structures minéralisées telles que l'os ou les dents. En revanche, les mesures ne sont pas réalisables sur les reconstructions 3D surfaciques $[5,21]$.

Pour la résolution en densité que le système permet d'afficher (échelle de niveaux de gris), elle est inférieure à celle du scanner (16 bits versus 12 bits). La TVFC est donc à limiter aux structures denses, telles que l'os et les dents.

La résolution de l'image dépend de plusieurs facteurs incluant la qualité et la résolution de l'écran plat du détecteur, et le nombre et l'espacement rotationnel des images de base de chaque individu à partir duquel le volume tridimensionnel a été généré [5].

D'autres facteurs peuvent affecter la résolution tels que la sophistication de l'algorithme de reconstruction dans le logiciel, la puissance de la source de rayons $X$ et la résolution du moniteur de visualisation [5].

L'augmentation de la résolution survient toujours avec une augmentation de la dose d'irradiation $[5,16]$.

\section{Rapidité d'acquisition}

La TVFC acquiert en général toutes les images en une seule rotation, ce qui réduit le temps d'acquisition à moins de 20 secondes. Chaque coupe tomodensitométrique peut nécessiter environ 10 secondes, alors que la TVFC peut balayer une tête entière en 10 seoondes. Ce temps réduit le mouvement du sujet, ce qui réduit les artefacts de mouvements [9].

Quant à la reconstruction, elle doit être effectuée dans un délai acceptable (moins de 5 minutes). Ce temps de reconstruction dépend des paramètres d'acquisition (taille du voxel, field of view, nombre de projections), du hardware (rapidité de traitement, débit des données de l'acquisition jusqu'au poste de travail de l'ordinateur), et du logiciel (reconstruction d'algorithmes) utilisés $[6,9]$.

\section{Champ de vue (FOV) variable}

La capacité de contrôler le FOV est exceptionnellement importante en termes de limitation de la dose des rayons $X[5,16]$. Plus le champ de vue est variable, plus la dose de rayons $X$ est faible. Cette limitation respecte donc davantage les organes radiosensibles tels que la cornée, les glandes salivaires ou la thyroïde [9].

Un FOV optimal peut être sélectionné pour chaque patient selon la pathologie ou la région à radiographier $[9,16]$. Si un champ limité est suffisant pour les indications courantes de l'imagerie 3D (implantologie, rapports de dents incluses avec les structures avoisinantes), les bilans orthodontiques et la chirurgie maxillofaciale exigent des champs plus grands [7].

\section{Facilité d'utilisation et analyse en temps réel}

La reconstruction des données de la TVFC peut être faite normalement dans un ordinateur. La manipulation de ces outils est à la portée de tous, ce qui rend l'analyse des données et des 
images facile et procure même la possibilité de faire une analyse en temps réel.

Contrairement à la TVFC, les images du scanner se présentent souvent imprimées et pour les visualiser sur un ordinateur sous un format adapté, elles nécessitent beaucoup de temps et

\section{Limites de la TVFC}

À part le coût d'achat qui reste élevé, la TVFC a très peu d'inconvénients.

Les inconvénients inhérents à cette technique sont en relation avec ses propres avantages et, en particulier, avec les faibles doses de rayons $X$ délivrées.

La réduction des doses délivrées entraîne une augmentation du bruit au niveau de l'image, et donc, d'une réduction de sa qualité.

La résolution spatiale peut être moins élevée comparée à l'examen tomodensitométrique, particulièrement pour une dosimétrie faible ou un champ de vue limité, entraînant une précision géométrique moins fine, mais amplement suffisante dans les diverses indications dentaires et maxillofaciales.

L'apparition de différents artefacts, que ce soit des artefacts de mouvement ou de haute densité, constitue l'un des principaux inconvénients de l'imagerie volumique.

Les artefacts de mouvement sont les plus fréquents en tomographie volumétrique numéri-

\section{Conclusion}

La technique d'imagerie la plus appropriée est celle qui fournit le plus d'informations, qui peut d'argent, ce qui retarde la phase de diagnostic $[6,9]$.

Enfin, les appareils cone beam nécessitent peu d'espace (comparable à celui d'un orthopantomogramme) et peuvent ainsi être facilement installés dans un cabinet dentaire [5].

sée. Ils sont provoqués, la plupart du temps, par les mouvements de déglutition, les mouvements respiratoires ou de bascule de la tête. La prévention suppose une information préalable délivrée au patient qui devra rester immobile entre 36 et 75 secondes selon I'appareil utilisé, soit la durée totale de l'examen, en réduisant au maximum les mouvements décrits.

Cet aspect limite l'indication de cet examen chez les enfants en âge de comprendre et de mettre en application ces consignes $[6,21]$.

Concernant les artefacts de haute densité générés par d'éventuelles restaurations métalliques ou des appareillages orthodontiques fixes, ils sont très nettement réduits comparés à ceux générés par le scanner. En effet, ce dernier utilise un faisceau de rayons $X$ beaucoup plus puissant, associé à des détecteurs à sensibilité plus importante $[6,21]$.

Enfin, la TVFC reste limitée à l'exploration des tissus dentaires et osseux et donne peu d'informations sur les tissus mous [6].

influencer la qualité des soins au patient, et pour une dosimétrie la plus faible possible. 
Le cone beam doit de ce fait respecter :

- le principe de justification (le bénéfice doit être supérieur au risque potentiel) ;

- le principe d'optimisation par lequel « l'exposition des personnes aux rayonnements ionisants doit être maintenue au niveau le plus faible qu'il est possible d'atteindre » (principe ALARA) pour un même résultat $[4,7]$.

Avec la diminution du coût de la TVFC, sa capacité diagnostique combinée à sa dose d'irradiation réduite, la TVFC devrait révolutionner la pratique de la chirurgie dentaire et maxillofaciale $[4,14]$.
Toutefois, la TVFC ne peut remplacer la radiographie endobuccale pour les applications générales de la médecine dentaire. Elle n'est qu'un complément et permet, grâce à ses avantages significatifs, de réduire le besoin du scanner conventionnel dans des applications dentaires et maxillofaciales.

À ce jour, des études basées sur l'evidence based sont encore nécessaires pour apprécier davantage l'apport de la tomographie volumique numérisée dans certaines indications, ainsi que pour établir des recommandations sur la meilleure façon d'utiliser le cone beam en imagerie dento-maxillofaciale.

\section{Bibliographie}

1. Bellaiche N. Scanner et tomographie à faisceau conique. Quelle méthode d'imagerie choisir en odontostomatologie? Le Fil Dentaire 2007;27:16-28.

2. Bjerklin $\mathrm{K}$, Ericson $\mathrm{S}$. How a computerized tomography examination changed the treatment plans of $\mathbf{8 0}$ children with retained and ectopically positioned maxillary canines. Angle Orthod 2006;76(1):43-51.

3. Bernardes RA, Gomes de Moraes IG, Húngaro Duarte MA, Azevedo BC, de Azevedo JR, Bramante $C$ Use of cone-beam volumetric tomography in the diagnosis of root fractures. Oral Surg Oral Med Oral
Pathol Oral Radiol Endod 2009;108(2):270-7.

4. Cavézian R. Imagerie dentomaxillaire : passé, présent, limites et perspectives. Les Journées Dentaires Internationales du Québec, 2008.

5. Dawood A, Patel S, Brown J. Cone beam CT in dental practice. Br Dental J 2009;207(1):23-8.

6. De Vos W, Casselman J, Swennen GR.

Cone-beam computerized tomography (CBCT) imaging of the oral and maxillofacial region: a systematic review of the literature. Int J Oral Maxillofac Surg 2009;38:609-25.

7. EADMFR (European Academy Dentomaxillofacial Radiology).
Basic principles for use of dental Cone Beam CT. Consensus guidelines of the European Academy of Dental and Maxillofacial Radiology.

January, 2009.

8. Éthier J, Forest D. Les applications de la tomographie volumique à faisceau conique (TVFC) en médecine dentaire. Journal de l'Ordre des Dentistes du Québec 2009;46(3):7-24.

9. Farman AG, Scarfe WC. The basics of maxillofacial Cone Beam Computed Tomography. In: Farman AG, Scarfe WC, Haskell BS. Guest eds. Cone Beam Computer Tomography.

Seminars in Orthodontics, ed. Sadowsky PL. Amsterdam : Elsevier Science B.V., 
The Netherlands

2009;15(1):2-13

10. Haney E, Gansky SA, Lee JS, Johnson E, Maki K, Miller AJ, Huang JC. Comparative analysis of traditional radiographs and cone-beam computed tomography volumetric images in the diagnosis and treatment planning of maxillary impacted canines.

Am J Orthod Dentofacial Orthop 2010;137(5):590-7.

11. HAS.

Tomographie volumique a faisceau conique de la face (cone beam computerized tomography).

Rapport d'évaluation technologique.

Saint-Denis, Décembre 2009.

12. HAS.

Tomographie volumique à faisceau conique de la face (cone beam). Note de cadrage. Saint-Denis, Juin 2009.

13. Lemkamp M, Filippi $A$, Berndt D, Lambrecht JT. Possibilités de diagnostic par tomographie volumique numérisée.

Rev Mens Suisse

Odontostomatol

2006;116(6):651-3.

14. Loubele $M$, Bogaerts $R$, Van Dijck E, Pauwels R, Vanheusden $S$, Suetens $P$, Marchal G, Sanderink G, Jacobs $\mathrm{R}$.

Comparison between effective radiation dose of CBCT and MSCT scanners for dentomaxillofacial applications. Eur J Radiol 2009;71(3):461-8.

15. Ludlow JB, Davies-Ludlow LE, Brooks SL, Howerton WB.
Dosimetry of 3 CBCT devices for oral and maxillofacial radiology: CB Mercuray, NewTom $3 G$ and i-CAT. Dentomaxillofac Radiol 2006;35(4):219-26.

16. MacDonald-Jankowski DS, Orpe EC.

Some current legal issues that may affect oral and maxillofacial radiology. Part 2: digital monitors and cone-beam computed tomography.

J Can Dent Assoc. 2007;73(6):507-11.

17. MacDonald-Jankowski DS, Orpe EC.

Computed tomography for oral and maxillofacial surgeons.

Part 2: Cone-beam computed tomography. Asian J Oral Maxillofac Surg 2006;18:85-92.

18. Neugebauer J, Shirani R, Mischkowski RA, Ritter L, Scheer M, Keeve E, Zöller JE. Comparison of cone-beam volumetric imaging and combined plain radiographs for localization of the mandibular canal before removal of impacted lower third molars.

Oral Surg Oral Med Oral Pathol Oral Radiol Endod 2008;105(5):633-42.

19. Ogawa N, Miyazaki $Y$, Kubota M, Huang JC, Miller AJ, Maki K.

Application of cone beam CT 3D images to cephalometric analysis. Orthodontic waves 2010;69(4):138-50.

20. Quereshy FA, Savell TA, Palomo JM.

Applications of cone beam computed tomography in the practice of oral and maxillofacial surgery.

J Oral Maxillofac Surg

2008;66(4):791-6.

21. Rouas $P$, Bandon $D$, Nancy J, Delbos $Y$, Hauret L.

La tomographie volumétrique numérisée par le système NewTom : intérêt de ce nouvel examen d'imagerie médicale chez l'enfant. Arch Pédiatr 2006;13:1169-77.

22. Suomalainen $A$, Ventä $I$, Mattila M, Turtola L, Vehmas T, Peltola JS. Reliability of CBCT and other radiographic methods in preoperative evaluation of lower third molars. Oral Surg Oral Med Oral Pathol Oral Radiol Endod 2010;109(2):276-84.

23. Tsiklakis K, Donta C, Gavala S, Karayianni K, Kamenopoulou V, Hourdakis CJ.

Dose reduction in maxillofacial imaging using low dose Cone Beam CT.

Eur J Radiol 2005;56(3):413-7.

24. Tyndall DA, Brooks SL. Selection criteria for dental implant site imaging: a position paper of the American Academy of Oral and Maxillofacial Radiology. Oral Surg Oral Med Oral Pathol Oral Radiol Endod 2000;89(5):630-7.

25. White SC, Pae EK. Patient image selection criteria for Cone Beam Computed Tomography imaging. Semin Orthod 2009;15:19-28. 


\section{SUMMARY}

\section{Cone beam computed tomography in dentomaxillofacial imaging}

Laila BENJELLOUN, Karima EL HARTI, Wafaa EL WADY

\section{Keywords \\ - cone beam computed tomography dentomaxillofacial imaging}

Cone beam computed tomography is a relatively new imaging technology that allows visualizing the complete maxillofacial complex.

Its technique, low dosimetry and its low price comparing with CT scanner permit to propose it as an alternative of the CT scanner, especially in some clinical applications such as odontogenic tumors, maxillofacial trauma, implantology and the relationship of third molars with the surrounding structures...

This promising technology is now widely diffused. It is therefore important to show the reel place of cone beam computed tomography in dentomaxillofacial imaging.

\section{Questions-Réponses}

>1. Quels sont les avantages de la tomographie volumique à faisceau conique par rapport à la tomodensitométrie ? Est-ce :

$\square$ a - la dose d'irradiation plus réduite ?

$\square \mathrm{b}$ - le temps d'examen plus réduit ?

$\square$ c - la possibilité d'explorer les tissus mous ?

$\square d$ - le coût réduit ?

'Ұ!npә, snןd иәшехә,p sduә ә - q

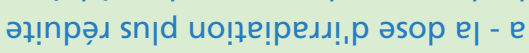

: ұuos səбеұuene sə| : əsuodọy
> 2. - Quelles sont les indications de la tomographie volumique à faisceau conique? :

a - bilan pré-implantaire ?

b - bilan radiologique des tumeurs vasculaires de la face ?

c - bilan radiologique des dents incluses ?

d - étude du rapport des dents de sagesse mandibulaires avec le canal dentaire inférieur?

¿גกว!ฺ

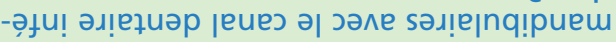

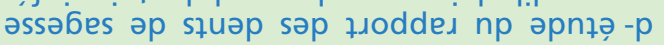
¿sәsnju! słuәp sәp әnb!̣о일 uеן!q ¿әл!етuе|du!-әرd ue!! -e : quos suo!̣eग!pu! sə] : əsuodə̧y 